\title{
Hot Topics in Research: Preventive Neuroradiology in Brain Aging and Cognitive Decline
}

\author{
C.A. Raji, H. Eyre, S.H. Wei, D.E. Bredesen, S. Moylan, M. Law, G. Small, P.M. Thompson, R.M. Friedlander, D.H. Silverman, B.T. Baune,
} T.A. Hoang, N. Salamon, A.W. Toga, and M.W. Vernooij

SUMMARY: Preventive neuroradiology is a new concept supported by growing literature. The main rationale of preventive neuroradiology is the application of multimodal brain imaging toward early and subclinical detection of brain disease and subsequent preventive actions through identification of modifiable risk factors. An insightful example of this is in the area of age-related cognitive decline, mild cognitive impairment, and dementia with potentially modifiable risk factors such as obesity, diet, sleep, hypertension, diabetes, depression, supplementation, smoking, and physical activity. In studying this link between lifestyle and cognitive decline, brain imaging markers may be instrumental as quantitative measures or even indicators of early disease. The purpose of this article is to provide an overview of the major studies reflecting how lifestyle factors affect the brain and cognition aging. In this hot topics review, we will specifically focus on obesity and physical activity.

ABBREVIATIONS: $\mathrm{AD}=$ Alzheimer disease $\mathrm{APOE}=$ Apolipoprotein $\mathrm{E} ; \mathrm{BMI}=$ body mass index; $\mathrm{MCl}=$ mild cognitive impairment

$\mathbf{P}$ reventive neuroradiology is an emerging discipline that has the potential to contribute substantially to human health. Preventive neuroradiology is the application of multimodal brain imaging toward early identification of subclinical brain disease prevention through the influence of modifiable risk factors. Positioned at the intersection of neurology and psychiatry, it has applications in a multitude of diseases including age-related cognitive decline, mood and psychotic disorders, and a variety of other neuropsychiatric conditions. Preventive neuroradiology also has application to the recently proposed field of health neuroscience, ${ }^{1}$ a concept that studies the interplay between the brain and physical health during the life span. ${ }^{1}$

A specific area where preventive neuroradiology is having an

Received February 15, 2015; accepted after revision February 23.

From the Departments of Radiology (C.A.R., S.H.W., T.A.H., N.S.), Psychiatry (H.E., G.S.), Neurology (D.E.B.), and Nuclear Medicine (D.H.S.), University of California at Los Angeles Medical Center, University of California at Los Angeles, Los Angeles, California; Discipline of Psychiatry (H.E., B.T.B.), University of Adelaide, Adelaide, South Australia, Australia; School of Medicine (S.M.), Deakin University, Geelong, Victoria, Australia; Department of Radiology (M.L.) and Laboratory of Neuroimaging (P.M.T., A.W.T.), University of Southern California, Los Angeles, California; Department of Neurosurgery (R.M.F.), University of Pittsburgh, Pittsburgh, Pennsylvania; and Departments of Radiology and Epidemiology (M.W.V.), Erasmus University Medical Center, Rotterdam, the Netherlands.

This work was supported by the National Institute of Biomedical Imaging and Bioengineering (grant P41EB015922) and the National Institutes of Health (grant U01AG024904).

Please address correspondence to Cyrus A. Raji, MD, PhD, Department of Radiology, UCLA Medical Center, 757 Westwood Blvd, Los Angeles, CA 90095; e-mail: cyrusraji@gmail.com; @cyrusraji

- Indicates open access to non-subscribers at www.ajnr.org

http://dx.doi.org/10.3174/ajnr.A4409 impact is in the area of age-related cognitive decline. As populations age, the burden of age-related cognitive decline is rising. Globally, the number of older individuals (60 years of age or older) is expected to more than double, from 841 million in 2013 to $>2$ billion in $2050,{ }^{2}$ with rates of dementia expected to increase from an estimated 44 million worldwide in $2013^{3}$ to an estimated 75.6 million in 2030, and 136 million in 2050 .

The expected precipitous rise in rates of age-related cognitive decline has not, to date, been met with a substantial expansion in treatment options. In fact, current pharmacologic therapies for the most common form of dementia, Alzheimer disease (AD), appear to provide some symptomatic relief but do not influence the underlying pathophysiology. ${ }^{4}$ This problem signifies the need to focus on increasing our understanding of preventive lifestyle modifications and their neurobiologic underpinnings of the disease. For example, with $\mathrm{AD}$, a recent study has highlighted the important role of risk-factor reduction for decreasing prevalence. ${ }^{5}$ Barnes and Yaffe $^{5}$ explored the role of 7 potentially modifiable AD risk factors: diabetes, midlife hypertension, midlife obesity, smoking, depression, low educational attainment, and physical inactivity. They determined that these factors contributed to up to half of AD cases globally (17.2 million), and a 10\%-25\% reduction in all risk factors could potentially prevent as many as 1.1-3.0 million cases. An emerging literature currently explores the effect of these risk factors on neurobiology factors. Other lifestyle factors that have been found to reduce the risk of $\mathrm{AD}$ include mind-body exercise (eg, yoga, tai chi, qi gong $^{6}$ ), conventional physical activity (eg, aerobic, strength training $^{6}$ ), nutritional supplements (eg, $\omega$-3 fatty acids, flavonols ${ }^{7}$ ), stressreduction techniques (eg, Mindfulness-Based Stress Reduction ${ }^{8}$ ), 
sleep-modification strategies, ${ }^{9}$ and dietary interventions (eg, fish consumption, Mediterranean diet). ${ }^{10}$

Classic risk-factor studies in dementia are hampered by the difficult phenotyping of the disease with large heterogeneity in severity and presentation, and by the fact that once symptomatic, irreversible brain damage is already present. The paradigm shift in medicine from cure toward prevention calls for identification of potentially modifiable factors that affect disease in an earlier stage. During the past decades, brain imaging has proved instrumental as a means of studying brain changes in aging and age-related cognitive decline. ${ }^{1-14}$ Many new imaging markers have been identified that either serve as quantifiable intermediate factors in the pathway of lifestyle and age-related cognitive decline or could even indicate disease in an early stage. For example, the hippocampal structure has been shown to be affected both positively and negatively by lifestyle factors by using structural, functional, and molecular forms of neuroimaging. ${ }^{15,16}$ Neuroradiology as a discipline is hence highly important in better understanding the role of these lifestyle factors in positively or negatively affecting structural, functional, and molecular radiologic correlates.

In this hot topics in research review, we explore the potential role for "preventive neuroradiology" in the risk reduction of neuropsychiatric disorders, by using age-related cognitive decline as an example.

\section{OBESITY AND AGE-RELATED COGNITIVE DECLINE Insights from Clinical Trials and Epidemiology}

There is evidence of an association between midlife obesity and increased risk of dementia. The Barnes and Yaffe model ${ }^{5}$ suggests a pooled relative risk estimate of 1.60 (95\% CI, 1.34-1.92) and approximately $2 \%(677,000)$ of $\mathrm{AD}$ cases worldwide are potentially attributable to midlife obesity. A recent systematic review ${ }^{17}$ identified 10 prospective studies that examined the association between various measures of body weight and dementia, of which 7 were suitable for inclusion in a meta-analysis. Three of 4 studies found that body mass index (BMI) (as a continuous measure) was associated with an increased risk of all-cause dementia; 2 of 5 studies indicated that obesity $(\mathrm{BMI} \geq 30)$ was associated with an increased risk of all-cause dementia; and 2 of 5 studies found that obesity was associated with an increased risk for $\mathrm{AD}$.

\section{Insights from Neurobiology}

Commonly proposed mediators for the relationship between higher body tissue adiposity and brain structure at any age include hypercortisolemia, reduced exercise, impaired respiratory function, inflammation, oxidative stress, cardiovascular disease, hypertension and/or hyperlipidemia, and type 2 diabetes mellitus. ${ }^{18,19}$ The co-occurrence of at least 3 of the following cardiovascular factors including large waist circumference (or adiposity), increased triglycerides, elevated blood pressure, and fasting hyperglycemia has been referred to as "the metabolic syndrome." ${ }^{20}$ Adiposity is also associated with insulin resistance and subsequent type 2 diabetes, hypertension, dyslipidemia, cardiovascular disease, degenerative joint disease, cancer, and lung disease. ${ }^{21}$ Moreover, an elevated BMI is significantly correlated with a reduction in neuronal fiber bundle length, which is believed to contribute to brain atro- phy. Finally, greater brain atrophy may occur in people with central leptin insufficiency, a marker of obesity. ${ }^{18}$

\section{Insights from Neuroradiology: Structural Imaging}

Volumetric MR Imaging. Several studies have investigated the relationship between BMI and brain atrophy. In an early study, ${ }^{18}$ tensor-based morphometry was used to examine gray matter and white matter volume differences in 94 elderly subjects who remained cognitively healthy for at least 5 years after their scan. When controlling for age, sex, and race, subjects with obesity with a high BMI (BMI of >30) showed atrophy in the frontal lobes, anterior cingulate gyrus, hippocampus, and thalamus compared with individuals with a normal BMI (18.5-25). Overweight subjects (BMI of 25-30) had atrophy in the basal ganglia and corona radiata of the WM. Overall brain volume did not differ between overweight and obese persons. In a similar study, Bobb et $\mathrm{al}^{22}$ explored 5-year longitudinal associations between change in BMI and gray matter with time, in a cohort of 247 former heavy-metal workers and community controls. Higher baseline BMI was associated with greater decline in temporal and occipital GM ROI volumes. Change in BMI during the 5-year period was only associated with a change in hippocampal volume and not other ROIs. Overall, higher BMI was associated with declines in volume in temporal and occipital GM with time.

A recent study ${ }^{23}$ explored the associations among physical activity, BMI, and brain structure in normal aging, mild cognitive impairment (MCI), and AD dementia. This study included 963 participants from the multisite Cardiovascular Health Study including healthy controls $(n=724)$, patients with Alzheimer dementia $(n=104)$, and individuals with MCI $(n=135)$. Physical activity was independently associated with greater whole-brain and regional brain volumes and reduced ventricular dilation. Those with higher BMI had lower whole-brain and regional brain volumes. A physical activity-BMI conjunction analysis showed brain preservation with physical activity and volume loss with increased BMI in overlapping brain regions (Fig 1).

A study by Driscoll et $\mathrm{al}^{24}$ explored the influence of midlife obesity on brain atrophy. In this study, associations between global (BMI) and central (waist circumference) midlife obesity and subsequent trajectories of regional brain atrophy in 152 individuals $[\mathrm{M}$ (age) $=69 \pm 7.8$ years] were examined. After followup, 21 individuals became cognitively impaired. Results suggest that midlife obesity may be an important modifier of brain atrophy in individuals who are developing cognitive impairment and dementia, while it has little effect on structural brain integrity in older adults without dementia.

Brain Tissue Integrity. MR imaging advancements such as diffusion tensor imaging have more recently been used to quantify subtle changes in WM tract microstructure and connectivity in senescent and developing adolescent brains. Furthermore, the use of tractography and diffusion pattern statistical analyses is instrumental in determining the spatial definition of individual tracts. Research has shown that cerebral WM integrity is decreased in the aging brain, particularly affecting the prefrontal regions. ${ }^{25}$

A recent study by Stanek et $\mathrm{al}^{26}$ examined the association between BMI and WM integrity. The authors used a diffusion 


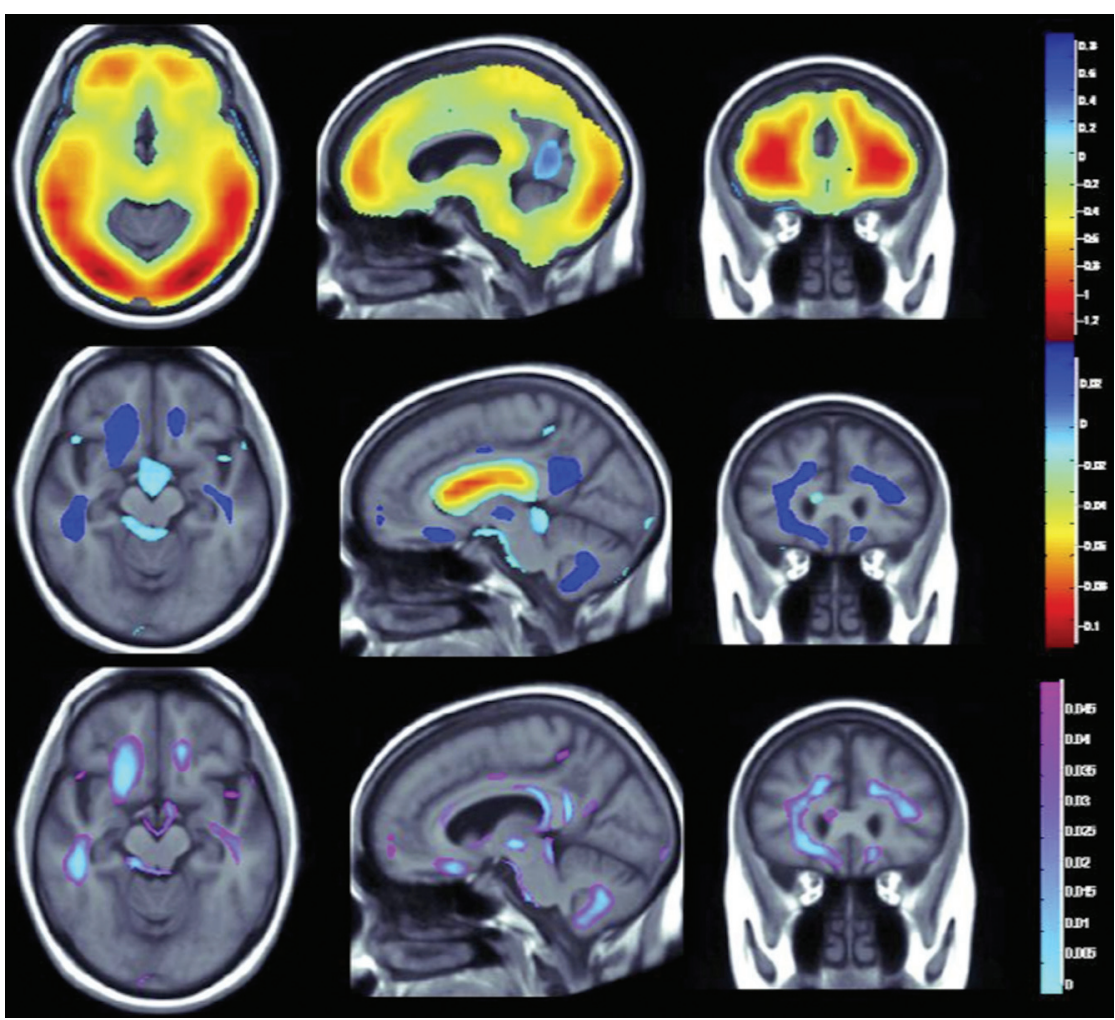

FIG 1. Relationship between obesity and physical activity on brain structure (adapted with permission from Boyle et $\mathrm{a}^{23}$ ). The top panel shows whole-brain 3D maps in which regional brain volumes are inversely correlated with BMI. The middle panel shows where regional brain volumes positively correlate with physical activity. The lower panel shows regions of significant brain volume differences from both higher BMI and physical activity in a conjunction analysis that identifies brain areas that are independently influenced by these factors.

tensor imaging index of tract coherence-fractional anisotropy to examine the structure of the corpus callosum and fornix. This study classified 103 adult participants between 21 and 86 years of age without a history of neurologic, medical, or psychiatric illness according to BMI. Individuals with obesity demonstrated lower fractional anisotropy than healthy and overweight persons for all WM indexes, but no fractional anisotropy differences emerged between overweight and healthy individuals.

Functional Imaging. Functional MR imaging may provide an efficacious method for assessing the impact of adiposity in cognitively intact middle-aged adults because it can identify altered brain activation patterns indicative of cognitive vulnerability. ${ }^{27}$ Prior work has identified changes in brain activation during cognition in association with elevated BMI. ${ }^{28-30}$ One recent study explored how a centralized distribution of adiposity relates to brain activation during a working memory task in a cognitively intact middle-aged sample. ${ }^{31}$ Seventy-three adults, $40-60$ years of age, completed a verbal working memory task during fMRI. Central adiposity was assessed with waist circumference. Larger waist circumference was associated with a diminished working memory-related blood oxygen level-dependent response in the right superior frontal gyrus and left middle frontal gyrus. Reduced task-related activation in the right superior frontal gyrus and left middle frontal gyrus was related to slower reaction time on the task, controlling for age and education.
PHYSICAL ACTIVITY AND AGERELATED COGNITIVE DECLINE Insights from Clinical Trials and Epidemiology

Conventional physical activity is one of the most promising therapies for the treatment and prevention of age-related cognitive decline and dementia. ${ }^{4}$ Most important, there are a variety of subtypes of physical activity, from aerobic activity, to resistance, to stretching and toning. A recent randomized controlled trial $^{32}$ examined the efficacy of resistance and aerobic training in the improvement of cognitive functions in subjects with subjective MCI. The study occurred during 6 months and involved 86 community-dwelling women 70-80 years of age. Physical activity protocols included twice-weekly resistance training, twice-weekly aerobic training, or twice-weekly balance and toning training (ie, control). Resistance training improved selective attention/conflict resolution and associated memory compared with balance and toning training. In contrast, aerobic physical activity improved general balance and mobility and cardiovascular capacity. The study also found that aerobic physical activity improved verbal memory and both resistance and aerobic physical activity improved spatial memory.

A recent meta-analysis ${ }^{33}$ examined the efficacy of exercise on cognition in older adults with MCI. MCI was diagnosed based on documented criteria or via the Mini-Mental State Examination. Fourteen randomized controlled trials with 1695 participants $65-95$ years of age were used. These studies had a duration of $6-52$ weeks. Overall, $42 \%$ of effect sizes were potentially clinically relevant (effect size of $>0.20$ ) with only $8 \%$ of cognitive outcomes statistically significant. The meta-analysis revealed negligible but significant effects of exercise on verbal fluency (effect size: 0.17 [range, $0.04-0.30]$ ). No significant benefit was found for additional executive measures, memory, or information processing. The authors critically appraised randomized controlled trial methods and concluded that they had moderate quality, with most trial samples being too small for sufficient power. They suggested that there is clearly some effect of exercise at moderate-tohigh levels of exertion.

Physical activity is on a continuum with physical inactivity. Worldwide, approximately $13 \%$ (nearly 4.3 million) of AD cases may be attributable to physical inactivity. ${ }^{5}$ A recent systematic review and meta-analysis identified 16 prospective studies on the association between physical activity and dementia that included 163,797 older adults without dementia at baseline and 3219 individuals with dementia at follow-up. ${ }^{34}$ The combined relative risk in the highest-versus-lowest physical activity groups was 0.72 for

AINR Am J Neuroradiol 36:1803-09 Oct 2015 wwwajnr.org 1805 
all-cause dementia and 0.55 for AD. The previously mentioned Barnes and Yaffe ${ }^{5}$ model suggests a relative risk of 1.82 for AD.

\section{Insights from Neurobiology}

The biologic mechanisms by which cognition is enhanced through physical exercise remain to be completely elucidated, though the number of studies that have tried to identify these mechanisms has increased in the past 10 years. For the most part, the studies that support the notion that physical exercise has an impact on brain functions have focused on the direct biologic effects of exercise by using both animal and human models. ${ }^{6}$ However, exercise may enhance cognition indirectly by improving health conditions (ie, stress, sleep), reducing chronic diseases (ie, coronary heart disease) that impact neurocognitive functions, and offering psychological and social effects. ${ }^{35}$ The molecular mechanisms by which exercise induces angiogenesis, neurogenesis, and synaptogenesis have received growing attention in recent years. $^{6}$

From a mechanistic perspective, sedentary behaviors may contribute to the risk of $\mathrm{AD}$ and dementia by 2 main factors. ${ }^{5,36}$ First, they are associated with an increased risk of cardiometabolic risk factors - diabetes, hypertension, obesity ${ }^{37}$ — that are associated with an increased risk of dementia. ${ }^{38,39}$ Second, sedentary behaviors appear to have direct effects on neurobiologic processes. A recent review ${ }^{40}$ outlines evidence to suggest that sedentary behaviors may have detrimental effects on the brain via reducing neurogenesis, synaptic plasticity, neurotrophin production, and angiogenesis and by increasing inflammation. Most important however, no studies have integrated objective measurements of both sedentary behavior and physical activity with measures of cognition or potential mechanistic outcomes such as neurogenesis and synaptic plasticity, regional fat deposits including visceral fat and pericardial fat, disrupted glucose metabolism, and/or inflammation. ${ }^{40}$

\section{Insights from Neuroradiology: Structural Brain Imaging}

Volumetric MR Imaging. Imaging studies corroborate the findings of the beneficial effects of exercise on brain structure and function. Higher gray and white matter cortical volumes were present on MR volumetric imaging in individuals with and without dementia with better aerobic fitness (maximal oxygen consumption, maximal oxygen uptake, peak oxygen uptake, or maximal aerobic capacity). ${ }^{41,42} \mathrm{MR}$ imaging studies in elderly populations without dementia also found that bilateral hippocampal volumes are better maintained in fit individuals, with resultant superior performance in tasks related to spatial memory. ${ }^{43}$ A cross-sectional study by Makizako et al ${ }^{44}$ examined associations between light- and moderate-intensity physical activity (measured with accelerometers), total duration of physical activity, hippocampal volume, and memory in older adults with MCI. Moderate physical activity was associated with hippocampal volume after controlling for age, but light and total physical activity were not. Both light and moderate physical activity groups were not associated with memory performance. Structural equation modeling demonstrated that moderate physical activity was not directly associated with memory but significantly contributed to hippocampal volume; hippocampal volume loss was significantly and directly associated with poor memory performance. This finding suggests that the benefits of moderate physical activity on memory among older adults with MCI may be mediated by hippocampal volume. Most intriguing, reversal of age-related hippocampal volume loss by $2 \%$, equivalent to $1-2$ years of volume loss, was observed after exercise training during late adulthood; this finding was accompanied by a concomitant increased serum brain-derived neurotropic factor and improved memory function. ${ }^{45}$

Randomized controlled trials have demonstrated the neuroprotective effects of exercise on brain aging in elderly populations, including in those with dementia, leading to positive effects on brain structure and cognitive performance. Some studies also suggested that these effects may be dependent on the duration, quality, and variety of exercise. ${ }^{46}$

No studies have examined the relationship between sedentary behavior and brain structure. ${ }^{40}$ We hypothesize that sedentary behaviors are associated with atrophy of numerous brain regions, particularly the hippocampus. Faster rates of hippocampal volume decay are associated with MCI and $\mathrm{AD} .{ }^{47,48}$

Functional Imaging. We are aware of 1 study exploring hippocampal physiology (blood flow) in sedentary older adults. ${ }^{49}$ In this study, investigators used resting hippocampal cerebral blood flow measures (via arterial spin-labeling MR imaging) and sedentary time/physical activity (via accelerometry) on 33 cognitively healthy adults (52-81 years of age), 9 of whom were Apolipoprotein $E(A P O E) \varepsilon 4$ carriers. Results indicated that the relationship between sedentary time and cerebral blood flow in the left hippocampus differs by $A P O E$ status, whereby $A P O E \varepsilon 4$ carriers show higher cerebral blood flow as a function of longer sedentary time compared with noncarriers, possibly suggesting a cerebral blood flow regulatory response to compensate for metabolic alterations in dementia risk. These data suggest that the relationship between cerebral blood flow and sedentary time is different in $A P O E \varepsilon 4$ carriers and noncarriers and that sedentary time may act as a behavioral risk factor for cerebral blood flow dysregulation in those at genetic risk for developing AD.

Types of physical activity other than cardiovascular have also been shown to help improve cognitive function. Strength training frequency of at least once per week was associated with greater cerebrovascular perfusion in older women compared with those who those who did not engage in strength-training activities. ${ }^{50} \mathrm{~A}$ 12-month long, twice-weekly resistance training program in elderly women found functional changes in the regions of the cerebral cortex, most strikingly in the left middle temporal gyrus and the left anterior insula to the lateral orbital frontal cortex, associated with improved hemodynamics and response inhibition based on the Flanker test performance. ${ }^{51}$ In addition, a 12-month randomized trial of exercise, including both cardiovascular and coordination training, in older adults showed that improved motor fitness was associated with retained or improved hippocampal volumes. $^{52}$

Brain Tissue Integrity. Life-long aerobic fitness in a small number of elderly athletes was also associated with findings of reduced white matter hyperintensities by $83 \%$ compared with sedentary individuals and higher fractional anisotropy within multiple 
white matter tracts related to motor control and coordination. ${ }^{53}$ White matter hyperintensities are a marker of vascular damage. Active exercise in the elderly is associated with lower mean diffusivity in the cingulate cortex and medial temporal lobe, suggestive of improved microstructural integrity within memory and executive function networks. ${ }^{54}$ A 1-year exercise program showed a promising association between improved fitness and increased white matter integrity in the frontal and temporal lobes and improved short-term memory, though the cognitive improvements were not independently associated with imaging findings. ${ }^{55} \mathrm{Im}$ provements in temporal lobe connectivity are associated with changes in levels of serum growth factors such as brain-derived neurotropic factor, vascular endothelial growth factor, and insulin-like growth factor-1; these molecules may serve as biomarkers for neurologic function and may underlie the biochemical regulation of white matter plasticity. ${ }^{46,56}$

\section{Molecular Imaging}

Emerging evidence suggests that higher levels of physical exercise/ fitness are associated with reduced amyloid $\mathrm{A} \beta$ burden $^{57-59}$ and potentially lowered intracellular $\tau$ protein $^{58}$; however, there are some null findings. ${ }^{60,61}$ A recent study ${ }^{59}$ aimed to see if plasma $A \beta$ and $\mathrm{A} \beta$ brain deposition were associated with physical activity levels and whether these associations differed between carriers and noncarriers of the APOE $\varepsilon 4$ allele. Five-hundred forty-six cognitively intact participants (60-95 years of age) were included in these analyses, with physical activity assessed by questionnaire. A subgroup ( $n=116$ ) underwent ${ }^{11} \mathrm{C}$ Pittsburgh compound-B positron-emission tomography scanning to quantify brain amyloid load. After stratification of the cohort based on APOE $\varepsilon 4$ allele carriage, it was evident that only noncarriers received the benefit of reduced plasma $\mathrm{A} \beta$ from physical activity. Conversely, lower levels of Pittsburgh compound-B were observed in higher exercising $A P O E \varepsilon 4$ carriers. Lower plasma A $\beta 1-42 / 1-40$ and brain amyloid were observed in those reporting higher levels of physical activity.

Another recent study ${ }^{62}$ explored whether engagement in physical activity might favorably alter the age-dependent evolution of $\mathrm{AD}$-related brain and cognitive changes in a cohort of at-risk, late-middle-aged adults. In this study, 317 enrollees underwent an MR imaging acquisition; a subset also underwent ${ }^{11} \mathrm{C}$ Pittsburgh compound-B PET $(n=186)$ and $\left[{ }^{18} \mathrm{~F}\right]$ fluorodeoxyglucose PET $(n=152)$. There were significant age $\times$ physical activity interactions for $\mathrm{A} \beta$ burden, glucose metabolism, and hippocampus volume. This finding suggested that with advancing age, physically active individuals exhibited a lesser degree of biomarker alterations compared with the physically inactive ones.

\section{DISCUSSION}

\section{Summary}

Overall, the results from this work highly suggest that the effects of lifestyle factors on cognitive function in aging act through impact on brain structure and function. Neuroimaging studies increasingly reveal structural and functional brain changes in relation to lifestyle factors that translate to measurable cognitive differences. This finding implies that these quantitative neuroimaging metrics may be effective surrogate markers that can be utilized in trials or can help identify novel pathways for intervention.

\section{Clinical and Public Health Implications}

There are some more immediate clinical implications for this field. Two relevant areas include rapid, automated quantitation of brain volume. ${ }^{63}$ This provides a relatively inexpensive means to track brain atrophy and the effects of lifestyle interventions; the lower cost compared with analysis by neuroradiologists may make this more available to the community. Additionally, the need for lifestyle modification advice and interventions for adult and late-life individuals is highly relevant to this field, given their noted effects on the brain. The global mental health implications of neuroscience were more broadly outlined recently. ${ }^{64}$

\section{Future Directions}

In this review, we have focused on obesity and physical activity and their effects on the brain. We are aware of a number of other lifestyle factors that require investigation across the neuroimaging modalities. For example, more recent studies have begun to parse out the potential effects of aerobic, resistance, and coordinative exercises and dual-task interventions in improving structural, functional, and cognitive aspects of aging. ${ }^{65}$ Other lifestyle factors that may positively affect the brain include mind-body therapies (eg, yoga, qi gong, tai $\mathrm{chi}^{6}$ ), supplements (eg, $\omega$-3 fatty acids, ${ }^{66}$ flavonols ${ }^{67}$ ), stress-reduction techniques (eg, Mindfulness-Based Stress Reduction ${ }^{6}$ ), sleep-modification strategies, ${ }^{9}$ and dietary interventions (eg, fish consumption, ${ }^{15}$ Mediterranean $\operatorname{diet}^{68}$ ). Other lifestyle factors that impact the brain include smoking, alcohol consumption, sleep, and hypertension. Continuing this work is critical for the development of treatment plans and may affect health care policy and personal philosophies on preventive and therapeutic lifestyle modification. By taking a multifaceted approach to lifestyle modification, one may reap additive or synergistic benefits, even at an early age.

Future studies may help elucidate the mechanism underlying cognitive improvements and identify target populations in which a longitudinal regimen of nonpharmacologic lifestyle modifications may yield significant health benefits. Furthermore, the lifestyle modification may be tailored to individuals with specific illnesses or genetic predispositions with a feasible, preventive goal in mind. Most important, there are coexisting lifestyle factors and a relative lack of premorbid conditions associated with a healthy lifestyle that may act as confounds. These must be carefully considered in future studies.

Developing literature is exploring the role of preventive neuroradiology in other psychiatric disorders. In depression, a metaanalysis confirmed a direct correlation between increasing depressive episode numbers and decreased hippocampal volume. ${ }^{69}$ The hippocampus is, therefore, a potential target for preventive interventions. In schizophrenia, a group of ultra-high-risk subjects was followed longitudinally with structural MR imaging. ${ }^{70}$ This study found both groups of subjects who subsequently developed psychosis (schizophrenia and affective psychosis) showed reductions in the frontal cortex relative to ultra-high-risk subjects who did not develop psychosis. The subgroup that subsequently developed schizophrenia also showed smaller volumes in the pa- 
rietal cortex and, at trend level, in the temporal cortex, whereas those who developed an affective psychosis had significantly smaller subgenual cingulate volumes. This finding also raises targets for preventive interventions.

In preventive neuroradiology, imaging doctors act as actionable information consultants to both referring physicians and their patients in delivering the added value of quantitative neuroimaging. For example, if a neuroradiologist can provide quantitative measurements of hippocampal volume to a geriatric psychiatrist, thereby identifying significant hippocampal volume decrease in a patient with subtle memory loss, it gives that referring physician improved confidence as to the potential etiology of patient symptoms and subsequent management. Such actionable information can also be used to engage patients in brain-directed lifestyle programs that can then be reassessed with a combination of clinical evaluations and follow-up quantitative neuroimaging. ${ }^{71}$ In such a new clinic model, neuroradiologists can act as visible team members for improving patient outcomes. This aspect of preventive neuroradiology is also an illustrative example of value-based imaging that is promoted as important for the future of radiology. ${ }^{72}$

Disclosures: Cyrus A. Raji-UNRELATED: Consultancy: Brainreader ApS, Change Your Brain Change Your Life Foundation. Dale E. Bredsen—UNRELATED: Employment: Muses Labs. Steven Moylan—UNRELATED: Employment: Barwon Health (clinical psychiatrist work); Other: Deakin University, Comments: employed as a clinical lecturer. Meng Law-RELATED: Grant: Toshiba*; Consulting Fee or Honorarium: Guebert; Consultancy: Bracco.* Gary Small—UNRELATED: Consultancy: Forum Pharmaceuticals, ${ }^{*}$ Herbalife, ${ }^{*}$ Novartis, ${ }^{*}$ Lilly, ${ }^{*}$ Pfizer,, Janssen*; Grants/Grants Pending: Pom Wonderful*; Payment for Lectures (including service on Speakers Bureaus): Novartis*; Patents (planned, pending or issued): FDDNP-PET; Royalties: HarperCollins, Workman Publishing Company, NewsMax Media; Stock/Stock Options: TauMark. Paul M. Thompson-RELATED: Grant: National Institutes of Health grants. * Dan H. Silverman-OTHER RELATIONSHIPS: Dr Dan Silverman is the inventor of NeuroQ, which is now licensed and sold separately by SynterMed (http:// www.syntermed.com/). Bernhard T. Baune-UNRELATED: Board Membership: Lundbeck; Employment: University of Adelaide; Grants/Grants Pending: National Health and Medical Research Council Australia; Payment for Lectures (including service on Speakers Bureaus): Lundbeck. Arthur W. Toga-RELATED: Grant: National Institute of Biomedical Imaging and Bioengineering, ${ }^{*}$ National Institutes of Health,* Comments: Laboratory of Neuro Imaging-Resource, Award No. P41EB015922; The Alzheimer's Disease Neuroimaging Initiative, Award No. U01AG024904. *Money paid to the institution.

\section{REFERENCES}

1. Erickson KI, Creswell JD, Verstynen TD, et al. Health neuroscience: defining a new field. Curr Dir Psychol Sci 2014;23:446-53

2. United Nations, Department of Economic and Social Affairs, Population Division. World Population Ageing 2013. ST/ESA/SER.A/348. New York: United Nations; 2013

3. Prince M, Prina M, Guerchet M; Alzheimer's Disease International. World Alzheimer Report 2013: Journey of Caring-An Analysis of LongTerm Care for Dementia. London: Alzheimer's Disease International; 2013

4. Selkoe DJ. Preventing Alzheimer's disease. Science 2012;337:1488-92

5. Barnes DE, Yaffe K. The projected effect of risk factor reduction on Alzheimer's disease prevalence. Lancet Neurol 2011;10:819-28

6. Eyre HA, Baune BT. Assessing for unique immunomodulatory and neuroplastic profiles of physical activity subtypes: a focus on psychiatric disorders. Brain Behav Immun 2014;39:42-55

7. Varteresian T, Lavretsky H. Natural products and supplements for geriatric depression and cognitive disorders: an evaluation of the research. Curr Psychiatry Rep 2014;16:456

8. Wells RE, Kerr CE, Wolkin J, et al. Meditation for adults with mild cognitive impairment: a pilot randomized trial. J Am Geriatr Soc 2013;61:642-45

9. Coogan AN, Schutová B, Husung S, et al. The circadian system in Alzheimer's disease: disturbances, mechanisms, and opportunities. Biol Psychiatry 2013;74:333-39

10. Di Marco LY, Marzo A, Muñoz-Ruiz M, et al. Modifiable lifestyle factors in dementia: a systematic review of longitudinal observational cohort studies. J Alzheimers Dis 2014;42:119-35

11. Hua X, Leow AD, Parikshak N, et al; Alzheimer's Disease Neuroimaging Initiative. Tensor-based morphometry as a neuroimaging biomarker for Alzheimer's disease: an MRI study of $676 \mathrm{AD}, \mathrm{MCI}$, and normal subjects. Neuroimage 2008;43:458-69

12. Mueller SG, Weiner MW, Thal LJ, et al. The Alzheimer's Disease Neuroimaging Initiative. Neuroimaging Clin N Am 2005;15:869-77, xi-xii

13. DeCarli C, Mungas D, Harvey D, et al. Memory impairment, but not cerebrovascular disease, predicts progression of MCI to dementia. Neurology 2004;63:220-27

14. Morra JH, Tu Z, Apostolova LG, et al; Alzheimer's Disease Neuroimaging Initiative. Automated mapping of hippocampal atrophy in 1-year repeat MRI data from 490 subjects with Alzheimer's disease, mild cognitive impairment, and elderly controls. Neuroimage 2009;45:S3-15

15. Raji CA, Erickson KI, Lopez OL, et al. Regular fish consumption and age-related brain gray matter loss. Am J Prev Med 2014;47:444-51

16. Ho AJ, Raji CA, Becker JT, et al. The effects of physical activity, education, and body mass index on the aging brain. Hum Brain Mapp 2011;32:1371-82

17. Beydoun MA, Beydoun HA, Wang Y. Obesity and central obesity as risk factors for incident dementia and its subtypes: a systematic review and meta-analysis. Obes Rev 2008;9:204-18

18. Raji CA, Ho AJ, Parikshak NN, et al. Brain structure and obesity. Hum Brain Mapp 2010;31:353-64

19. Moylan S, Berk M, Dean OM, et al. Oxidative \& nitrosative stress in depression: why so much stress? Neurosci Biobehav Rev 2014;45:46-62

20. Yaffe K, Haan M, Blackwell T, et al. Metabolic syndrome and cognitive decline in elderly Latinos: findings from the Sacramento Area Latino Study of Aging study. J Am Geriatr Soc 2007;55:758-62

21. Poirier P, Després JP, Bertrand OF. Identifying which patients with diabetes should be tested for the presence of coronary artery disease-the importance of baseline electrocardiogram and exercise testing. Can J Cardiol 2006;22(suppl A):9A-15A

22. Bobb JF, Schwartz BS, Davatzikos C, et al. Cross-sectional and longitudinal association of body mass index and brain volume. Hum Brain Mapp 2014;35:75-88

23. Boyle CP, Raji CA, Erickson KI, et al. Physical activity, body mass index, and brain atrophy in Alzheimer's disease. Neurobiol Aging 2015;36(suppl 1):S194-202

24. Driscoll I, Beydoun MA, An Y, et al. Midlife obesity and trajectories of brain volume changes in older adults. Hum Brain Mapp 2012;33:2204-10

25. Marks BL, Madden DJ, Bucur B, et al. Role of aerobic fitness and aging on cerebral white matter integrity. Ann N Y Acad Sci 2007;1097:171-74

26. Stanek KM, Grieve SM, Brickman AM, et al. Obesity is associated with reduced white matter integrity in otherwise healthy adults. Obesity 2011;19:500-04

27. Bondi MW, Houston WS, Eyler LT, et al. fMRI evidence of compensatory mechanisms in older adults at genetic risk for Alzheimer disease. Neurology 2005;64:501-08

28. Braskie MN, Small GW, Bookheimer SY. Vascular health risks and fMRI activation during a memory task in older adults. Neurobiol Aging 2010;31:1532-42

29. Gonzales MM, Tarumi T, Miles SC, et al. Insulin sensitivity as a mediator of the relationship between BMI and working memoryrelated brain activation. Obesity 2010;18:2131-37

30. Volkow ND, Wang GJ, Telang F, et al. Inverse association between 
BMI and prefrontal metabolic activity in healthy adults. Obesity 2009; 17:60-65

31. Gonzales MM, Kaur S, Eagan DE, et al. Central adiposity and the functional magnetic resonance imaging response to cognitive challenge. Int J Obes (Lond) 2014;38:1193-99

32. Nagamatsu LS, Handy TC, Hsu CL, et al. Resistance training promotes cognitive and functional brain plasticity in seniors with probable mild cognitive impairment. Arch Intern Med 2012; 172:666-68

33. Gates N, Fiatarone Singh MA, Sachdev PS, et al. The effect of exercise training on cognitive function in older adults with mild cognitive impairment: a meta-analysis of randomized controlled trials. $\mathrm{Am} \mathrm{J}$ Geriatr Psychiatry 2013;21:1086-97

34. Hamer M, Chida Y. Physical activity and risk of neurodegenerative disease: a systematic review of prospective evidence. Psychol Med 2009;39:3-11

35. Bherer L, Erickson KI, Liu-Ambrose T. A review of the effects of physical activity and exercise on cognitive and brain functions in older adults. J Aging Res 2013;2013:657508

36. Barnes DE, Whitmer RA, Yaffe K. Physical activity and dementia: the need for prevention trials. Exerc Sport Sci Rev 2007;35:24-29

37. de Rezende LF, Rey-López JP, Matsudo VK, et al. Sedentary behavior and health outcomes among older adults: a systematic review. $B M C$ Public Health 2014;14:333

38. Profenno LA, Porsteinsson AP, Faraone SV. Meta-analysis of Alzheimer's disease risk with obesity, diabetes, and related disorders. Biol Psychiatry 2010;67:505-12

39. Kennelly SP, Lawlor BA, Kenny RA. Blood pressure and the risk for dementia: a double edged sword. Ageing Res Rev 2009;8:61-70

40. Voss MW, Carr LJ, Clark R, et al. Revenge of the "sit" II: does lifestyle impact neuronal and cognitive health through distinct mechanisms associated with sedentary behavior and physical activity? Ment Health Physical Act 2014;7:9-24

41. Colcombe SJ, Kramer AF, McAuley E, et al. Neurocognitive aging and cardiovascular fitness: recent findings and future directions. $J$ Mol Neurosci 2004;24:9-14

42. Colcombe SJ, Erickson KI, Scalf PE, et al. Aerobic exercise training increases brain volume in aging humans. J Gerontol A Biol Sci Med Sci 2006;61:1166-70

43. Erickson KI, Prakash RS, Voss MW, et al. Aerobic fitness is associated with hippocampal volume in elderly humans. Hippocampus 2009;19:1030-39

44. Makizako $\mathrm{H}$, Liu-Ambrose $\mathrm{T}$, Shimada $\mathrm{H}$, et al. Moderate-intensity physical activity, hippocampal volume, and memory in older adults with mild cognitive impairment. J Gerontol A Biol Sci Med Sci 2015;70:480-86

45. Erickson KI, Voss MW, Prakash RS, et al. Exercise training increases size of hippocampus and improves memory. Proc Natl Acad Sci U S A 2011;108:3017-22

46. Kirk-Sanchez NJ, McGough EL. Physical exercise and cognitive performance in the elderly: current perspectives. Clin Interv Aging 2014;9:51-62

47. Fein G, Di Sclafani V, Tanabe J, et al. Hippocampal and cortical atrophy predict dementia in subcortical ischemic vascular disease. Neurology 2000;55:1626-35

48. Mueller SG, Schuff N, Yaffe K, et al. Hippocampal atrophy patterns in mild cognitive impairment and Alzheimer's disease. Hum Brain Mapp 2010;31:1339-47

49. Zlatar ZZ, Wierenga CE, Bangen KJ, et al. Increased hippocampal blood flow in sedentary older adults at genetic risk for Alzheimer's disease. J Alzheimers Dis 2014;41:809-17

50. Xu X, Jerskey BA, Cote DM, et al. Cerebrovascular perfusion among older adults is moderated by strength training and gender. Neurosci Lett 2014;560:26-30

51. Liu-Ambrose T, Nagamatsu LS, Voss MW, et al. Resistance training and functional plasticity of the aging brain: a 12-month randomized controlled trial. Neurobiol Aging 2012;33:1690-98

52. Niemann C, Godde B, Voelcker-Rehage C. Not only cardiovascular, but also coordinative exercise increases hippocampal volume in older adults. Front Aging Neurosci 2014;6:170

53. Tseng BY, Gundapuneedi T, Khan MA, et al. White matter integrity in physically fit older adults. Neuroimage 2013;82:510-16

54. Tian Q, Erickson KI, Simonsick EM, et al. Physical activity predicts microstructural integrity in memory-related networks in very old adults. J Gerontol A Biol Sci Med Sci 2014;69:1284-90

55. Voss MW, Heo S, Prakash RS, et al. The influence of aerobic fitness on cerebral white matter integrity and cognitive function in older adults: results of a one-year exercise intervention. Hum Brain Mapp 2013;34:2972-85

56. Voss MW, Erickson KI, Prakash RS, et al. Neurobiological markers of exercise-related brain plasticity in older adults. Brain Behav Immun 2013;28:90-99

57. Head D, Bugg JM, Goate AM, et al. Exercise engagement as a moderator of the effects of APOE genotype on amyloid deposition. Arch Neurol 2012;69:636-43

58. Liang KY, Mintun MA, Fagan AM, et al. Exercise and Alzheimer's disease biomarkers in cognitively normal older adults. Ann Neurol 2010;68:311-18

59. Brown BM, Peiffer JJ, Taddei K, et al. Physical activity and amyloid-â plasma and brain levels: results from the Australian Imaging, Biomarkers and Lifestyle Study of Ageing. Mol Psychiatry 2013;18:875-81

60. Vemuri P, Lesnick TG, Przybelski SA, et al. Effect of lifestyle activities on Alzheimer disease biomarkers and cognition. Ann Neurol 2012;72:730-38

61. Bugg JM, Head D. Exercise moderates age-related atrophy of the medial temporal lobe. Neurobiol Aging 2011;32:506-14

62. Okonkwo OC, Schultz SA, Oh JM, et al. Physical activity attenuates age-related biomarker alterations in preclinical AD. Neurology 2014;83:1753-60

63. Rathakrishnan BG, Doraiswamy PM, Petrella JR. Science to practice: translating automated brain MRI volumetry in Alzheimer's disease from research to routine diagnostic use in the work-up of dementia. Front Neurol 2014;4:216

64. Stein DJ, He Y, Phillips A, et al. Global mental health and neuroscience: potential synergies. Lancet Psychiatry 2015;2:178-85

65. Gregory MA, Gill DP, Petrella RJ. Brain health and exercise in older adults. Curr Sports Med Rep 2013;12:256-71

66. Cederholm T, Salem N Jr, Palmblad J. $\boldsymbol{\omega}$-3 fatty acids in the prevention of cognitive decline in humans. Adv Nutr 2013;4:672-76

67. Brickman AM, Khan UA, Provenzano FA, et al. Enhancing dentate gyrus function with dietary flavanols improves cognition in older adults. Nat Neurosci 2014;17:1798-803

68. Mosconi L, Murray J, Tsui WH, et al. Mediterranean diet and magnetic resonance imaging-assessed brain atrophy in cognitively normal individuals at risk for Alzheimer's disease. J Prev of Alzheimers Dis 2014;1:23-32

69. Videbech P, Ravnkilde B. Hippocampal volume and depression: a meta-analysis of MRI studies. Am J Psychiatry 2004;161:1957-66

70. Dazzan P, Soulsby B, Mechelli A, et al. Volumetric abnormalities predating the onset of schizophrenia and affective psychoses: an MRI study in subjects at ultrahigh risk of psychosis. Schizophr Bull 2012;38:1083-91

71. MacIntosh BJ, Swardfager W, Crane DE, et al. Cardiopulmonary fitness correlates with regional cerebral grey matter perfusion and density in men with coronary artery disease. PLoS One 2014;9: e91251

72. Norbash A, Bluth E, Lee CI, et al. Radiologist manpower considerations and Imaging 3.0: effort planning for value-based imaging. J Am Coll Radiol 2014;11:953-58 